\title{
ON THE STRUCTURE OF COMAXIMAL GRAPHS OF COMMUTATIVE RINGS WITH IDENTITY
}

\author{
SLAVKO M. MOCONJA ${ }^{\bowtie}$ and ZORAN Z. PETROVIĆ
}

(Received 3 November 2009)

\begin{abstract}
In this paper we investigate the center, radius and girth of comaximal graphs of commutative rings. We also provide some counterexamples to the results concerning the relation between isomorphisms of comaximal graphs and the rings in question. In addition, we investigate the relation between the comaximal graph of a ring and its subrings of a certain type.
\end{abstract}

2010 Mathematics subject classification: primary 13A15; secondary 05C75.

Keywords and phrases: commutative rings, maximal ideals, comaximal graph.

\section{Introduction}

In [2] Beck introduced zero-divisor graphs of commutative rings with identity. This concept was later extended to the case of noncommutative rings (see [6]). On the other hand, the concept of the comaximal graph for commutative rings with identity was introduced in [7] (without in fact being given a name) and in [3] further properties of these graphs were established.

Let us first record a few notions from graph theory which will be used in this paper. For a graph $\Gamma$, we denote by $E(\Gamma)$ and $V(\Gamma)$ the set of all edges and vertices, respectively. We recall that a graph is connected if there exists a path connecting any two distinct vertices. The distance between two distinct vertices $a$ and $b$, denoted by $d(a, b)$, is the length of the shortest path connecting them (if such a path does not exist, then $d(a, b)=\infty)$. The diameter of a graph $\Gamma$, denoted by $\operatorname{diam}(\Gamma)$, is equal to

$$
\sup \{d(a, b) \mid a, b \in V(\Gamma)\} .
$$

The girth of a graph $\Gamma$, denoted by $g(\Gamma)$, is the length of the shortest cycle in $\Gamma$. The eccentricity of a vertex $a$ is defined as a $\sup \{d(a, x): x \in V(\Gamma)\}$. If the diameter of a graph is finite, it is interesting to see what is the smallest eccentricity of a vertex in $\Gamma$. This is called the radius of the graph, and the vertices of $\Gamma$ with this smallest eccentricity form the center of the graph.

This work was partially supported by Ministry of Science and Environmental Protection of Republic of Serbia Project \#144018 and Project \#144020.

(C) 2010 Australian Mathematical Publishing Association Inc. 0004-9727/2010 \$16.00 
The coloring of a graph is an assignment of colors to vertices of the graph in such a way that adjacent vertices are assigned different colors. A graph is $n$-colorable if it is possible to give such a coloring with $n$ colors. The chromatic number of a graph $\Gamma$, denoted by $\chi(\Gamma)$, is the smallest $n$ such that the graph $\Gamma$ is $n$-colorable. If such an $n$ does not exist (which means that it is not possible to color this graph with only finitely many colors), one puts $\chi(\Gamma)=\infty$.

A subset $S \subseteq V(\Gamma)$ is a clique if the subgraph generated by this set is complete.

Finally, let us define a blow-up of a graph (see [4] —we give a more general definition). Let $\Gamma$ be a graph and $\left(\kappa_{x}\right)_{x \in V(\Gamma)}$ be a collection of nonzero cardinals. We denote by $\Gamma\left(\left(\kappa_{x}\right)_{x \in V(\Gamma)}\right)$ the graph which we get from $\Gamma$ by replacing any vertex $x$ of $\Gamma$ by a set $V_{x}$ of cardinality $\kappa_{x}$ and any edge $\{x, y\} \in E(\Gamma)$ by a complete bipartite graph whose vertex classes are $V_{x}$ and $V_{y}$. Note that two blow-ups $\Gamma\left(\left(\kappa_{x}\right)_{x \in V(\Gamma)}\right)$ and $\Gamma\left(\left(\kappa_{x}^{\prime}\right)_{x \in V(\Gamma)}\right)$ of the same graph $\Gamma$ are isomorphic if $\kappa_{x}=\kappa_{x}^{\prime}$ for all $x \in V(\Gamma)$.

Let $R$ be a commutative ring with identity. The comaximal graph $\Gamma(R)$ is defined as follows:

$$
V(\Gamma(R))=R, \quad E(\Gamma(R))=\left\{\{a, b\} \in[R]^{2}: R a+R b=R\right\},
$$

where, for a set $X$, we denote by $[X]^{2}$ the set of all two-element subsets of $X$. Let us denote by $\Gamma_{1}(R)$ the subgraph spanned by units, by $\Gamma_{2}(R)$ the subgraph spanned by all other elements (see [3]), and by $\Gamma_{2}^{\prime}(R)$ the subgraph of $\Gamma_{2}(R)$ spanned by elements not belonging to the Jacobson radical $\mathrm{J}(R)$.

In [7] the main result is that $\chi(\Gamma(R))$ is finite if and only if the ring $R$ itself is finite. In [3] the authors showed that the subgraph $\Gamma_{2}^{\prime}(R)$ is always connected, with diameter at most 3, that it is a complete bipartite graph if and only if it contains exactly two maximal ideals (if it contains $n$ maximal ideals then it is $n$-partite), gave the necessary and sufficient conditions for a diameter of this graph to be 2 , and showed that for some types of rings the fact that $\Gamma(R) \cong \Gamma(S)$ implies that $R \cong S$.

The organization of this paper is as follows. In the second section, with the exception of the simple lemma at the beginning, we discuss the case of semilocal rings (rings with finitely many maximal ideals, not necessarily Noetherian). We are able to give the complete description of comaximal graphs in this case (see [3]). As a byproduct we show that the comaximal graph of semilocal rings is $n$-colorable if and only if it contains $n$ maximal ideals.

In the third section we tackle the case of rings with infinitely many maximal ideals. We show that in this case comaximal graph contains an infinite clique and therefore cannot be finitely colorable. Conjointly with results from the previous section we get a generalization of the main result of [7]. We also discuss the center and radius of this graph. In particular, we show that the radius is equal to 2 for Dedekind domains. At the end of this section, we also give a result concerning the ring derived from its comaximal graph.

In the short fourth section we discuss a counterexample related to the results of Section 4 in [3]. 
The fifth section is dedicated to the relation between the comaximal graph of a ring and its subrings which are closed with respect to taking the inverse.

Some of the results from this paper were announced in [5].

\section{Semilocal rings}

For $x \in R$, let us denote by $M(x)$ the set of all maximal ideals containing the element $x$. Directly from the definition, we can give a simple characterization.

LEMMA 2.1. The elements $a$ and $b$ are adjacent in the comaximal graph if and only if there does not exist a maximal ideal that contains both of them, that is,

$$
\{a, b\} \in E(\Gamma(R)) \Longleftrightarrow M(a) \cap M(b)=\emptyset .
$$

PROOF. If $a, b \in \mathfrak{m}$, where $\mathfrak{m}$ is some maximal ideal, then $R a+R b \subseteq \mathfrak{m}$, hence $a$ and $b$ are not adjacent. On the other hand, if $a$ and $b$ are not adjacent, then $R a+R b$ is a proper ideal of $R$, hence is contained in some maximal ideal $\mathfrak{m}$, and therefore $M(a) \cap M(b) \neq \emptyset$.

It is clear that any element in $\mathrm{U}(R)$ (invertible elements of $R$ ) is adjacent to any other element of $R$. Also, any element in $\mathrm{J}(R)$ is not adjacent to any other element from $R \backslash \mathrm{U}(R)$. Therefore, it is of great interest to investigate $\Gamma_{2}^{\prime}(R)$.

Let us denote by $\operatorname{Max}(R)$ the set of all maximal ideals of the ring $R$ (all the rings are commutative with identity and $1 \neq 0$ ). In this section we assume that our ring $R$ is semilocal, so

$$
\operatorname{Max}(R)=\left\{\mathfrak{m}_{1}, \ldots, \mathfrak{m}_{n}\right\}
$$

for some $n \in \mathbb{N}$. Let $S \subseteq\{1, \ldots, n\}$ denotes any subset of the set of indices of maximal ideals. We denote by $M_{S}\left(M^{S}\right)$ the intersection (union) of all maximal ideals whose indices belong to $S$ :

$$
M_{S}=\bigcap_{i \in S} \mathfrak{m}_{i}, \quad M^{S}=\bigcup_{i \in S} \mathfrak{m}_{i}
$$

LEMMA 2.2. $M_{S} \subseteq M_{T}$ if and only if $S \supseteq T$.

PROOF. Of course, one part of this equivalence is trivial. We only prove the nontrivial part. So, let us assume that $M_{S} \subseteq M_{T}$. If $t \in T$, then $M_{S} \subseteq M_{T} \subseteq \mathfrak{m}_{t}$ and it follows that $\mathfrak{m}_{s} \subseteq \mathfrak{m}_{t}$ for some $s \in S$ (for this and similar other results concerning basic properties of commutative rings the reader may, for example, consult [1]). Since these are maximal ideals, we must have $\mathfrak{m}_{s}=\mathfrak{m}_{t}$ and it follows that $t \in S$. Therefore, $T \subseteq S$ and we are done.

PROPOSITION 2.3. Let the notation be as above. The sets

$$
V_{S}=M_{S} \cap\left(M^{S^{c}}\right)^{c},
$$

for $S \subseteq\{1, \ldots, n\}$, form a decomposition of $R$ by nonempty sets. 
PROOF. It is clear that the union of the sets in question is equal to $R$ and that different ones are disjoint. What we really need to prove is that all the sets in question are actually nonempty. So, assume to the contrary that $V_{S}=\emptyset$ for some $S \subseteq\{1, \ldots, n\}$. Then

$$
M_{S} \subseteq M^{S^{c}}
$$

Since we are dealing with maximal ideals, we conclude that $M_{S} \subseteq \mathfrak{m}_{j}$ for some $j \notin S$, which is impossible by Lemma 2.2.

The previous proposition allows us to give a complete description of $\Gamma(R)$ in the case where $\operatorname{Max}(R)$ is finite. Namely, the sets $V_{S}$ consist of elements which belong exactly to maximal ideals with indices in $S$. In addition, any element of $V_{S}$ is connected by an edge to any element of $V_{T}$ if and only if $S \cap T=\emptyset$. Therefore, it is convenient to think not only of the graph $\Gamma(R)$ but also of the auxiliary graph $\mathbb{G}_{n}$ whose vertices are subsets of the set $\{1, \ldots, n\}$ and where two vertices are joined by an edge if and only if the sets in question are disjoint. We get the graph $\Gamma(R)$ from the auxiliary graph $\mathbb{G}_{n}$ by replacing any vertex of that graph by a nonempty set, while preserving the previously connected vertices. For $\Gamma_{2}^{\prime}(R)$ the auxiliary graph $\mathbb{G}_{n}^{\prime}$ is a subgraph of $\mathbb{G}_{n}$ spanned by proper nonempty subsets. For example, in the case where we have exactly two maximal ideals our auxiliary graph $\mathbb{G}_{2}^{\prime}$ for $\Gamma_{2}^{\prime}(R)$ reduces to two vertices and one edge. Therefore it is clear that the main graph is a complete bipartite graph. If we have exactly three maximal ideals we get for $\mathbb{G}_{3}^{\prime}$ six vertices and six edges forming a triangle with an additional edge attached to every vertex of that triangle and therefore the main graph is 3-partite, but cannot be complete 3-partite. The same holds for arbitrary $n$.

Using this description we are able to prove the following theorem.

TheOREM 2.4. Let $|\operatorname{Max}(R)|=n$. Then the following statements hold.

(1) The graph $\Gamma(R)\left(\Gamma_{2}^{\prime}(R)\right)$ is a blow-up of the graph $\mathbb{G}_{n}\left(\mathbb{G}_{n}^{\prime}\right)$.

(2) If $n \geq 2$ and $R \neq \mathbb{Z}_{2} \times F$, for a field $F$, the radius of the graph $\Gamma_{2}^{\prime}(R)$ is 2 and the center consists of elements belonging to exactly one maximal ideal.

(3) If $R \cong \mathbb{Z}_{2} \times F$, for a field $F$, the graph $\Gamma_{2}^{\prime}(R)$ is acyclic. For $n=2$ and $R \neq \mathbb{Z}_{2} \times F$ the girth of $\Gamma_{2}^{\prime}(R)$ is 4 , and for $n \geq 3$ the girth of this graph is 3 .

(4) $\chi\left(\Gamma_{2}^{\prime}(R)\right)=n$.

PROOF. (1) It is clear from the previous discussion that the graph $\Gamma(R)\left(\Gamma_{2}^{\prime}(R)\right)$ is a blow-up of the graph $\mathbb{G}_{n}\left(\mathbb{G}_{n}^{\prime}\right)$. Namely, since all the sets $V_{S}$ (for $S \subseteq\{1, \ldots, n\}$ ) are nonempty $\left(V_{\{1, \ldots, n\}}=\mathrm{J}(R), V_{\emptyset}=\mathrm{U}(R)\right)$, and an element from $V_{S}$ is adjacent to an element from $V_{T}$ if and only if $S \cap T=\emptyset$, we see that the claim is true.

Before proceeding further, let us remark that, for $x \in V_{S}$ and $y \in V_{T}$, the following statements hold:

(a) $\quad d(x, y)=1 \Longleftrightarrow S \cap T=\emptyset$;

(b) $\quad d(x, y)=2 \Longleftrightarrow S \cap T \neq \emptyset$ and $S \cup T \neq\{1, \ldots, n\}$;

(c) $\quad d(x, y)=3 \Longleftrightarrow S \cap T \neq \emptyset$ and $S \cup T=\{1, \ldots, n\}$. 
(2) If there exists an element $a$ such that $d(a, b)=1$ for all $b \neq a$, we see that $n=2$ and $V_{\{1\}}$ or $V_{\{2\}}$ is a singleton. This means that $R \cong \mathbb{Z}_{2} \times F$ (see [3]). So, let $a \in V_{\{i\}}$, be an arbitrary element which is contained only in the maximal ideal $\mathfrak{m}_{i}$, and $b$ any other element which is noninvertible and not in $\mathrm{J}(R)$ (say, $b \in V_{T}$ ). If $i \notin T$ then $a$ and $b$ are adjacent; if $i \in T$, then $d(a, b)=2$ (the distance cannot be 3 since $\{i\} \cup T=T \neq\{1, \ldots, n\})$. If $a \in V_{S}$, where $S$ has at least two elements, say $i$ and $j$, we see that, for any $b \in V_{\{j\}^{c}}, d(a, b)=3$ and the eccentricity of $a$ is greater than 2 . Therefore, the radius is 2 and the center is the union $\bigcup_{i \in\{1, \ldots, n\}} V_{\{i\}}$.

(3) If $n=2$ our graph $\Gamma_{2}^{\prime}(R)$ is a complete bipartite graph, and therefore the girth is 4 if both parts have more than one element. If one of these parts has one element we conclude that $R \cong \mathbb{Z}_{2} \times F$, and in this case our graph is acyclic. If $n \geq 3$, the graph contains a triangle.

(4) Actually, in [7] the authors showed that $\chi(\Gamma(R))=n+m$ where $n$ is the number of maximal ideals and $m$ is the number of invertible elements of a finite ring $R$. Basically the same method they used allows us to conclude that $\chi\left(\Gamma_{2}^{\prime}(R)\right)=n$, for any ring with exactly $n$ maximal ideals. First of all, it is clear that $\chi\left(\Gamma_{2}^{\prime}(R)\right)=\chi\left(\mathbb{G}_{n}^{\prime}\right)$. Therefore, one only needs to see what is the chromatic number of $\mathbb{G}_{n}^{\prime}$. Since this graph contains $K_{n}$ (complete graph with $n$ vertices), the chromatic number must be at least $n$. And actually, there is a coloring with $n$ colors: we use color 1 to color all proper subsets containing 1, color 2 to color all proper subsets which contain 2 but not 1 , color 3 to color all proper subsets which contain 3 but not 1 and 2, and so on.

\section{Infinitely many maximal ideals}

We assume now that $\operatorname{Max}(R)$ is infinite.

Proposition 3.1. There exists an element $a \in R \backslash(\mathrm{J}(R) \cup \mathrm{U}(R))$, such that $M(a)^{c}$ is infinite.

PROOF. Otherwise, any two elements in $R \backslash(\mathrm{J}(R) \cup \mathrm{U}(R))$ would be in all but finitely many maximal ideals, and since there are infinitely many maximal ideals, they would both belong to some maximal ideal, which contradicts the fact that $\Gamma_{2}^{\prime}(R)$ is connected (see [3, Theorem 3.1]).

PROPOSITION 3.2. Let $R$ be a ring with infinitely many maximal ideals. Further, let $a \in R \backslash(\mathrm{J}(R) \cup \mathrm{U}(R))$ be an element such that $M(a)^{c}$ is infinite. Then, there exists an element $b \in R \backslash(\mathrm{J}(R) \cup \mathrm{U}(R))$, such that $R a+R b=R$ and $M(a b)^{c}$ is infinite.

PROOF. Let $\mathfrak{m}_{1}$ and $\mathfrak{m}_{2}$ be two maximal ideals that do not contain $a$. Let $x$ be an element in $\mathfrak{m}_{2} \backslash \mathfrak{m}_{1}$. Then the element ax does not belong to $\mathfrak{m}_{1}$, hence there exists an element $y \in \mathfrak{m}_{1}$, such that $R a x+R y=R$, and therefore $R a+R y=R$, $y \in R \backslash(\mathrm{J}(R) \cup \mathrm{U}(R))$, and $a y \notin \mathfrak{m}_{2}$ (the last holds since $R x+R y=R$, so $y \notin \mathfrak{m}_{2}$ ).

If there are infinitely many maximal ideals not containing $a y$, we are done (take $b=y$ ). Otherwise, since $a y \notin \mathfrak{m}_{2}$, there exists $z \in \mathfrak{m}_{2}$ such that $R a y+R z=R$. Hence $a y$ and $z$ cannot belong to the same maximal ideal, and therefore $z$ is contained in only 
finitely many maximal ideals. Now we have $R a+R z=R, z \in R \backslash(\mathrm{J}(R) \cup \mathrm{U}(R))$ and, since there are infinitely many maximal ideals not containing $a$ and $z$ is contained in finitely many maximal ideals, there are infinitely many maximal ideals not containing $a z$. We conclude the proof by taking $b=z$.

THEOREM 3.3. The graph $\Gamma_{2}^{\prime}(R)$ contains an infinite clique if and only if $R$ has infinitely many maximal ideals.

Proof. Let $\Gamma_{2}^{\prime}(R)$ contain an infinite clique. Then, clearly, $R$ cannot have finitely many maximal ideals; otherwise if $R$ had at most $n$ maximal ideals, we could find in any $n+1$ elements of that clique two belonging to the same maximal ideal.

Let $R$ be a ring with infinitely many maximal ideals. Let $a_{0} \in R \backslash(\mathrm{J}(R) \cup \mathrm{U}(R))$, such that the set $M\left(a_{0}\right)^{c}$ is infinite. Let $b_{0}=a_{0}$ and let $a_{1}$ be an element in $R \backslash$ $(\mathrm{J}(R) \cup \mathrm{U}(R))$ such that $R b_{0}+R a_{1}=R$ and $M\left(b_{0} a_{1}\right)^{c}$ is infinite. Take $b_{1}=b_{0} a_{1}$. Proceeding in this way, we get elements $a_{0}, a_{1}, a_{2}, \ldots$ and $b_{0}, b_{1}, b_{2}, \ldots$ such that $b_{n}=\prod_{i=0}^{n} a_{n}$, and $R b_{n}+R a_{n+1}=R$, which shows that the elements $a_{0}, a_{1}, a_{2}, \ldots$ make an infinite clique in $\Gamma_{2}^{\prime}(R)$.

COROLlary 3.4. If $R$ has infinitely many maximal ideals, then the girth of $\Gamma_{2}^{\prime}(R)$ is 3 .

PROOF. Any three elements in an infinite clique make a cycle.

In [3, Proposition 2.3] the authors claim that $\Gamma_{2}^{\prime}(R)$ is $n$-partite if $R$ has $n$ maximal ideals. Also, they claim that if $\Gamma_{2}^{\prime}(R)$ is $n$-partite, then $R$ has at most $n$ maximal ideals, and if $\Gamma_{2}^{\prime}(R)$ is not $(n-1)$-partite, then $R$ has exactly $n$ maximal ideals. Unfortunately, the proof of this fact is incorrect (the chosen elements $x_{i}$ may also belong to some other maximal ideals, and therefore some of them might not be connected as claimed). This proof may easily be corrected in the case where the ring $R$ contains only finitely many maximal ideals, but such a proof does not go through in the case of infinitely many ideals. From this we can conclude, under the assumption that $R$ has finitely many maximal ideals, that the chromatic number of $\Gamma_{2}^{\prime}(R)$ is $n$ if and only if $R$ has $n$ maximal ideals. Theorem 3.3 completes this result.

COROLlARY 3.5. If $R$ has infinitely many maximal ideals, then $\Gamma_{2}^{\prime}(R)$ is not n-partite and not $n$-colorable, for any $n \in \mathbb{N}$.

PROOF. For the proof just take an infinite clique in $\Gamma_{2}^{\prime}(R)$. It cannot be finitely partitioned and is not finitely colorable.

Now let us discuss the radius of the graph $\Gamma_{2}^{\prime}(R)$ when $R$ has infinitely many maximal ideals. The following proposition gives some simple properties of the center.

PROPOSITION 3.6. If an element a belongs to the center, then

$$
\bigcup_{\mathfrak{m} \in M(a)} \mathfrak{m} \backslash \bigcup_{\mathfrak{m}^{\prime} \notin M(a)} \mathfrak{m}^{\prime}
$$


is a subset of the center. If a does not belong to the center, then $\bigcap_{\mathfrak{m} \in M(a)} \mathfrak{m}$ is disjoint with the center.

PROOF. Let us suppose that $a$ belongs to the center. It is clear that for any $b \in \bigcup_{\mathfrak{m} \in M(a)} \mathfrak{m} \backslash \bigcup_{\mathfrak{m} \notin M(a)} \mathfrak{m}$, the inclusion $M(b) \subseteq M(a)$ holds. Therefore if $a$ is adjacent to an element $c$, then so is $b$. The case where $a$ does not belong to the center follows from the previous one.

THEOREM 3.7. Let $S \subset \operatorname{Max}(R)$ be such that

$$
\bigcap_{\mathfrak{m} \in S} \mathfrak{m} \backslash \bigcup_{\mathfrak{m}^{\prime} \notin S} \mathfrak{m}^{\prime} \neq \emptyset,
$$

while, for every $T \subset S$,

$$
\bigcap_{\mathfrak{m} \in T} \mathfrak{m} \backslash \bigcup_{\mathfrak{m}^{\prime} \notin T} \mathfrak{m}^{\prime}=\emptyset .
$$

Then every element a such that $M(a)=S$ has eccentricity 2. Therefore, if such an $S$ exists then the radius of $\Gamma_{2}^{\prime}(R)$ is 2 and any element a such that $M(a)=S$ belongs to the center.

PROOF. Let $M(a)=S$ and suppose that $b \in R \backslash(\mathrm{J}(R) \cup \mathrm{U}(R))$ is an element not adjacent to $a$. Then $M(a) \cap M(b) \neq \emptyset$. If $a b \notin \mathrm{J}(R)$, then $a b \notin \mathfrak{m}$, for some maximal ideal $\mathfrak{m}$, and hence $R a b+\mathfrak{m}=R$, so there exists $c \in \mathfrak{m}$ such that $R a b+R c=R$, and therefore we have a path $a-c-b$, hence $d(a, b)=2$. If $a b \in \mathrm{J}(R)$, then $\operatorname{Max}(R) \backslash M(b) \subset M(a)$. If the set $S$ is a singleton, this cannot happen and we are done. Otherwise, since $b \notin \mathrm{J}(R)$, there is a maximal ideal from $M(a)$ not containing $b$, say $b \notin \mathfrak{m}_{0}$. But then $R b+\mathfrak{m}_{0}=R$, so there exists $d \in \mathfrak{m}_{0}$ such that $R b+R d=R$. Since $M(d) \cap M(b)=\emptyset$, we conclude that $M(d) \subset M(a)=S$ and

$$
d \in \bigcap_{\mathfrak{m} \in M(d)} \mathfrak{m} \backslash \bigcup_{\mathfrak{m}^{\prime} \notin M(d)} \mathfrak{m}^{\prime} .
$$

This contradiction concludes the proof.

COROLLARY 3.8. If there exists an element a such that $M(a)$ is finite, then the radius of the graph $\Gamma_{2}^{\prime}(R)$ is 2 .

PROOF. Let $S \subseteq M(a)$ be minimal among the nonempty subsets of $M(a)$ that satisfy property $(*)$ from Theorem 3.7. Since $M(a)$ is finite, such a subset always exists and we are done.

REMARK. From this corollary we can conclude that the radius of our graph is equal to 2 for all rings in which every element is contained in only finitely many maximal ideals - for example, in Dedekind domains. 
Proposition 3.9. For any element $a \in R \backslash(\mathrm{J}(R) \cup \mathrm{U}(R))$ with eccentricity 2, $M(a)^{c}$ is infinite.

Proof. Assume, to the contrary, that $\operatorname{Max}(R) \backslash M(a)=\left\{\mathfrak{m}_{1}, \ldots, \mathfrak{m}_{n}\right\}$. Let $\mathfrak{m}_{-1}$ and $\mathfrak{m}_{0}$ be two different maximal ideals in $M(a)$. We know that $\bigcap_{i=0}^{n} \mathfrak{m}_{n} \supseteq \mathrm{J}(R)$. If $\bigcap_{i=0}^{n} \mathfrak{m}_{n}=\mathrm{J}(R)$, then $\bigcap_{i=0}^{n} \mathfrak{m}_{n} \subseteq \mathfrak{m}_{-1}$, and therefore for some $0 \leq k \leq n, \mathfrak{m}_{k} \subseteq \mathfrak{m}_{-1}$, which is a contradiction. Hence, there exists $b \in \bigcap_{i=0}^{n} \mathfrak{m}_{n} \backslash \mathrm{J}(R)$. Since $a, b \in \mathfrak{m}_{0}, a$ and $b$ are not adjacent, hence $d(a, b)=2$. Therefore, we have a path $a-c-b$. Since $a$ and $c$ are adjacent, we conclude that $M(c) \subseteq\left\{\mathfrak{m}_{1}, \ldots, \mathfrak{m}_{n}\right\}$, but then $b$ and $c$ cannot be adjacent, which is a contradiction.

THEOREM 3.10. If $R$ is a ring with infinitely many maximal ideals, such that $\mathrm{J}(R)$ is prime, then for every element in $R \backslash(\mathrm{J}(R) \cup \mathrm{U}(R))$ there are infinitely many maximal ideals not containing it.

Proof. By [3, Proposition 3.3], the diameter of $\Gamma_{2}^{\prime}(R)$ is 2. Hence, Proposition 3.9 implies the proof.

\section{On isomorphisms of rings and graphs}

In the Section 4 of [3] the authors pose the question concerning the possibility that the existence of an isomorphism between comaximal graphs of certain rings implies the existence of an isomorphism of the rings themselves. Of course, it is not possible for this to hold in general and the authors provide examples illustrating this. They also prove that in some cases of finite rings $\Gamma(R) \cong \Gamma(S)$ implies $R \cong S$. In addition, they claim in their Proposition 4.7 that if $\Gamma(R) \cong \Gamma(S)$ then $R / \mathrm{J}(R) \cong S / \mathrm{J}(S)$. In the case where the rings $R$ and $S$ are finite, this result indeed follows from their Theorem 4.4, but in general this is not true and we provide an example showing this.

EXAMPLE 4.1. Let us denote by $\mathbb{Z}_{(p, q)}$ the following subring of $\mathbb{Q}$ :

$$
\mathbb{Z}_{(p, q)}=\left\{\frac{a}{b}: p \times b, q \times b\right\},
$$

for prime numbers $p$ and $q(p \neq q)$. This ring has exactly two maximal ideals: one generated by $p$ and the other generated by $q$. The Jacobson radical $\mathrm{J}\left(\mathbb{Z}_{(p, q)}\right)$ is the ideal generated by $p q$ and $\mathbb{Z}_{(p, q)} / \mathrm{J}\left(\mathbb{Z}_{(p, q)}\right) \cong \mathbb{Z}_{p q}$. Since the ring $\mathbb{Z}_{(p, q)}$ has exactly two maximal ideals the structure of $\Gamma\left(\mathbb{Z}_{(p, q)}\right)\left(\Gamma_{2}^{\prime}\left(\mathbb{Z}_{(p, q)}\right)\right)$ is very simple-it is a blowup of $\mathbb{G}_{2}\left(\mathbb{G}_{2}^{\prime}\right)$ in which all cardinals are $\aleph_{0}$.

In particular, this is true for any other set $\{r, s\}$ of prime numbers $r$ and $s$ such that $r \neq s$. Therefore $\Gamma\left(\mathbb{Z}_{(p, q)}\right) \cong \Gamma\left(\mathbb{Z}_{(r, s)}\right)\left(\Gamma_{2}^{\prime}\left(\mathbb{Z}_{(p, q)}\right) \cong \Gamma_{2}^{\prime}\left(\mathbb{Z}_{(r, s)}\right)\right)$ for all prime numbers $p, q, r, s(p \neq q, r \neq s)$ but the quotient rings $\mathbb{Z}_{p q} \cong \mathbb{Z}_{r s}$ are isomorphic only if $\mathbb{Z}_{(p, q)} \cong \mathbb{Z}_{(r, s)}$. If $\{p, q\} \neq\{r, s\}$ the graphs $\Gamma_{2}^{\prime}\left(\mathbb{Z}_{p q}\right)$ and $\Gamma_{2}^{\prime}\left(\mathbb{Z}_{r s}\right)$ are not isomorphic either: they are both complete bipartite graphs but the sizes of the parts do not match. 


\section{On a connection between comaximal graph of a subring and a group of units in a subring}

Let $S$ be a subring of a commutative ring with identity $R$ (so $1 \in S$ ). If $x \in \mathrm{U}(S$ ) then $x \in S$ is invertible in $R$, that is, $\mathrm{U}(R) \cap S \supseteq \mathrm{U}(S)$, while the other inclusion need not hold.

In [8] the authors investigate the assumptions for rings (not necessarily commutative) on which the equality

$$
\mathrm{U}(R) \cap S=\mathrm{U}(S)
$$

holds. For example, it does hold in the case where $R$ is a Noetherian $S$-module (see [8]).

Let $S$ be a subring of a ring $R$. We investigate the question whether

$$
E(\Gamma(R)) \cap[S]^{2}=E(\Gamma(S))
$$

holds. If we assume that for $a, b \in S,\{a, b\} \notin E(\Gamma(R))$, then we have that there is a maximal ideal $\mathfrak{m} \triangleleft R$ containing both $a$ and $b$. But then $a, b \in \mathfrak{m} \cap S \triangleleft S$, and there is a maximal ideal $\mathfrak{n} \triangleleft S$ containing $\mathfrak{m} \cap S$, so we get that $\{a, b\} \notin E(\Gamma(S))$. From this observation we see that $E(\Gamma(R)) \cap[S]^{2} \supseteq E(\Gamma(S))$ always holds, and it is easy to see that the other inclusion need not be true.

Proposition 5.1. Let $S$ be a subring of a ring $R$. If $E(\Gamma(R)) \cap[S]^{2}=E(\Gamma(S))$ is true then $\mathrm{U}(R) \cap S=\mathrm{U}(S)$ is true.

PROOF. Let $a \in S$ be invertible in $R$. Then $\{a, 0\} \in E(\Gamma(R))$ and by the condition $\{a, 0\} \in E(\Gamma(S))$. Hence $a \in \mathrm{U}(S)$.

Even if $\mathrm{U}(R) \cap S=\mathrm{U}(S)$ it may happen that $E(\Gamma(R)) \cap[S]^{2} \neq E(\Gamma(S))$.

EXAMPLE 5.2. Let $R$ be an integral domain. Let us observe the ring $R[X, Y]$ and its subring $R[X Y, Y]$. Since $\mathrm{U}(R[X, Y])=\mathrm{U}(R[X Y, Y])=\mathrm{U}(R)$, it is true that $\mathrm{U}(R[X, Y]) \cap R[X Y, Y]=\mathrm{U}(R[X Y, Y])$. The elements $1+X Y$ and $Y \in R[X Y, Y]$ are adjacent in $\Gamma(R[X, Y])$, since $(1+X Y)+(-X) Y=1$. We wish to prove that $1+X Y$ and $Y$ are not adjacent in $\Gamma(R[X Y, Y])$.

Let us assume that $f(X Y, Y)(1+X Y)+g(X Y, Y) Y=1$. We may write

$$
f(X Y, Y)=f_{0}(Y)+f_{1}(Y) X Y+\cdots+f_{n}(Y)(X Y)^{n}
$$

and

$$
g(X Y, Y)=g_{0}(Y)+g_{1}(Y) X Y+\cdots+g_{m}(Y)(X Y)^{m} .
$$

We thus obtain

$$
\begin{aligned}
& f_{0}(Y)+\left(f_{0}(Y)+f_{1}(Y)\right) X Y+\cdots+\left(f_{n-1}(Y)+f_{n}(Y)\right)(X Y)^{n} \\
& \quad+f_{n}(Y)(X Y)^{n+1}+g_{0}(Y) Y+g_{1}(Y) Y X Y+\cdots+g_{m}(Y) Y(X Y)^{m}=1 .
\end{aligned}
$$


Comparing the terms which do not contain $X$, we may conclude that $f_{0}(Y)=1+$ $Y \bar{f}_{0}(Y)$ and $g_{0}(Y)=-\bar{f}_{0}(Y)$, for some $\bar{f}_{0}(Y)$. Now we get

$$
\begin{aligned}
& \left(1+Y \bar{f}_{0}(Y)+f_{1}(Y)\right) X Y+\cdots+\left(f_{n-1}(Y)+f_{n}(Y)\right)(X Y)^{n} \\
& \quad+f_{n}(Y)(X Y)^{n+1}+g_{1}(Y) Y X Y+\cdots+g_{m}(Y) Y(X Y)^{m}=0 .
\end{aligned}
$$

When we divide by $Y$, this equation becomes

$$
\begin{gathered}
\left(1+Y \bar{f}_{0}(Y)+f_{1}(Y)\right) X+\cdots+\left(f_{n-1}(Y)+f_{n}(Y)\right) X^{n} Y^{n-1} \\
\quad+f_{n}(Y) X^{n+1} Y^{n}+g_{1}(Y) X Y+\cdots+g_{m}(Y)(X Y)^{m}=0
\end{gathered}
$$

The coefficient on the term $X^{n+1} Y^{n}$ is the constant term from $f_{n}(Y)$ and is zero. Further, by observing the coefficients on $X^{n} Y^{n-1}, \ldots, X^{2} Y$, we conclude that all constant terms in the polynomials $f_{n-1}(Y), \ldots, f_{1}(Y)$ are zero. Finally, we get that the coefficient on $X$ is 1 , so that term never cancels, which is not possible. We thus conclude that $1+X Y$ and $Y$ are not adjacent in $\Gamma(R[X Y, Y])$.

The next proposition shows that equality (5.2) holds in the case where $R$ is a semilocal ring.

PROPOSITION 5.3. Let $S$ be a subring of a semilocal ring $R$. Then $E(\Gamma(R)) \cap[S]^{2}=$ $E(\Gamma(S))$ if and only if $\mathrm{U}(R) \cap S=\mathrm{U}(S)$.

PROOF. We only need to prove that $\mathrm{U}(R) \cap S=\mathrm{U}(S)$ implies that $E(\Gamma(R)) \cap[S]^{2}=$ $E(\Gamma(S))$. Let $\operatorname{Max}(R)=\left\{\mathfrak{m}_{1}, \ldots, \mathfrak{m}_{n}\right\}$. Note that $R=\mathrm{U}(R) \cup \mathfrak{m}_{1} \cup \cdots \cup \mathfrak{m}_{n}$, so

$$
\begin{aligned}
S=R \cap S & =(\mathrm{U}(R) \cap S) \cup\left(\mathfrak{m}_{1} \cap S\right) \cup \cdots \cup\left(\mathfrak{m}_{n} \cap S\right) \\
& =\mathrm{U}(S) \cup\left(\mathfrak{m}_{1} \cap S\right) \cup \cdots \cup\left(\mathfrak{m}_{n} \cap S\right) .
\end{aligned}
$$

If $\mathfrak{n} \triangleleft S$ is a maximal ideal, then $\mathfrak{n} \subseteq\left(\mathfrak{m}_{1} \cap S\right) \cup \cdots \cup\left(\mathfrak{m}_{n} \cap S\right)$, and since all $\mathfrak{m}_{i} \cap S$ are prime (they are contractions of prime ideals $\mathfrak{m}_{i}$ in $R$ ), we conclude that $\mathfrak{n} \subseteq \mathfrak{m}_{i} \cap S$, for some $i$. Since $\mathfrak{n}$ is maximal, we have that $\mathfrak{n}=\mathfrak{m}_{i} \cap S$ (we use here some elementary facts about commutative rings, which can be found, for example, in [1]). Let us now assume that for $a, b \in S,\{a, b\} \in E(\Gamma(R))$. Then there are no maximal ideals in $R$ containing both $a$ and $b$. If we assume that there exists a maximal ideal in $S$ containing $a$ and $b$, say $\mathfrak{n}$, we have $\mathfrak{n}=\mathfrak{m}_{i} \cap S$, for some $i$ and $a, b \in \mathfrak{n} \subseteq \mathfrak{m}_{i}$, which is a contradiction. Thus $\{a, b\} \in E(\Gamma(S))$.

REMARK 5.4. Let us observe that the same argument will work in the case where $S$ has finitely many prime ideals.

The condition that $R$ is semilocal is not necessary, as the following example shows.

EXAMPLE 5.5. Let $R$ again be an integral domain, and let us observe the ring $R[X]$ and $R$ as its subring. The condition $\mathrm{U}(R[X]) \cap R=\mathrm{U}(R)$ is satisfied since $\mathrm{U}(R[X])=\mathrm{U}(R)$. Assume that elements $a, b \in R$ are adjacent in $\Gamma(R[X])$, that is, 
that there exist polynomials $f(X), g(X) \in R[X]$ such that $a f(X)+b g(X)=1$. If $f_{0}, g_{0} \in R$ are constant terms of these polynomials, then we have $a f_{0}+b g_{0}=1$, which means that $a$ and $b$ are adjacent in $\Gamma(R)$. So it is true that $E(\Gamma(R[X])) \cap[S]^{2}=$ $E(\Gamma(R))$ although the ring $R[X]$ may also contain infinitely many maximal ideals and its subring $R$ infinitely many prime ideals.

\section{References}

[1] M. F. Atiyah and I. G. Macdonald, Introduction to Commutative Algebra (Addison-Wesley, Reading, MA, 1969).

[2] I. Beck, 'Coloring of commutative rings', J. Algebra 116 (1988), 208-226.

[3] H. R. Maimani, M. Salimi, A. Sattari and S. Yassemi, 'Comaximal graph of commutative rings', J. Algebra 319 (2008), 1801-1808.

[4] V. Nikiforov, 'Graphs with many copies of a given subgraph', Electron. J. Combin. 15(1) (2008), \# N6.

[5] Z. Z. Petrović and S. M. Moconja, 'On graphs associated to rings', Novi Sad J. Math. 38(3) (2008), 33-38.

[6] S. Redmond, 'The zero-divisor graph of a non-commutative ring', Internat. J. Commutative Rings 1(4) (2002), 203-211.

[7] P. D. Sharma and S. M. Bhatwadekar, 'A note on graphical representation of rings', J. Algebra 176 (1995), 124-127.

[8] J. Szigeti and L. van Wyk, 'Subrings which are closed with respect to taking the inverse', J. Algebra 318 (2007), 1068-1076.

SLAVKO M. MOCONJA, University of Belgrade, Faculty of Mathematics,

Studentski trg 16, Belgrade 11000, Serbia

e-mail: slavko@matf.bg.ac.rs

ZORAN Z. PETROVIĆ, University of Belgrade, Faculty of Mathematics,

Studentski trg 16, Belgrade 11000, Serbia

e-mail: zoranp@matf.bg.ac.rs 